\title{
La importancia de la participación educativa en los procesos de mejora
}

\author{
The importance of educational participation in improvement \\ processes
}

\section{*Christian Danner Loyola Bustos}

Loyola, C. D. (2020). La importancia de la participación educativa en los procesos de mejora. Revista Convergencia Educativa, 7, julio, 1-18. DOI: http://doi.org/10.29035/rce.7.1

[Recibido: 30 marzo, 2020 / Aceptado: 18 abril, 2020]

\section{RESUMEN}

Participar en un establecimiento educacional es integrarse activamente en la comunidad escolar con el fin de aportar a un objetivo compartido por todos: el mejoramiento de la calidad de la educación. El presente estudio tiene como objetivo dar a conocer la importancia que tiene la participación en los procesos de mejora educativa a partir de la revisión de bibliografía especializada. Se analizará el rol de la política educativa en la promoción de la participación escolar, resaltando la Política Nacional de Convivencia Escolar y el Plan de Formación Ciudadana.

Palabras clave: participación educativa, mejora escolar, Política Nacional de Convivencia Escolar, formación ciudadana.

\begin{abstract}
To participate in an educational institution is to be actively integrated into the school community in order to contribute to a goal shared by all: the improvement of the quality of education. The present study aims to make known the importance of participation in the processes of educational improvement based on the review of specialized literature. The role of education policy in promoting school participation will be analysed, highlighting the National Policy for School Coexistence and the Citizen Training Plan.
\end{abstract}

Key words: Educational Participation, School Improvement, National School Coexistence Policy, Citizen Training.

\footnotetext{
* Pontificia Universidad Católica de Valparaíso PUCV, Santiago, Chile.
} 
Loyola, C. D. (2020). La importancia de la participación educativa en los procesos de mejora.

Revista Convergencia Educativa 7, julio, 1-18. DOI: http://doi.org/10.29035/rce.7.1

\section{INTRODUCCIÓN. LA IMPORTRANCIA DE LA PARTICIPACIÓN}

La participación asociada al mundo escolar es un concepto que se ha venido trabajando fuertemente en las diversas investigaciones educativas durante el último tiempo. Actualmente, la participación corresponde a un proceso fundamental para generar procesos de mejora educativa efectiva quedando demostrado en una serie de marcos normativos y legales de la política educativa. De acuerdo con un documento del Ministerio de Educación, "participar en un establecimiento educacional es integrarse activamente en la comunidad escolar con el fin de aportar a un objetivo compartido por todos: el mejoramiento de la calidad de la educación" (Mineduc, 2016, 3).

La participación incluye múltiples formas (instancias formales e informales) en que se puede ser un aporte, entendiéndose la participación como un proceso de involucramiento de los distintos actores de la comunidad escolar en las decisiones y acciones que los afectan a ellos o a su entorno. Esta puede darse de manera individual u organizada (Unicef, 2005, en Mineduc, 2016, 3).

En la Ley General Educación define la participación como un espacio donde "los miembros de la comunidad educativa tienen derechos a ser informados y participar en el proceso educativo en conformidad a la normativa vigente" (LGE, 2009, 4). Las visiones y expectativas que se tiene sobre la escuela se fortalecen cuando los líderes educativos promueven dinámicas en sus comunidades para desarrollar relaciones de apoyo mutuo y propósitos compartidos (Leithwood \& Riehl, 2003, citado en Mineduc, 2016).

\subsection{OBJETIVO Y PERTINENCIA}

El presente ensayo tiene como finalidad conocer e identificar algunas características propias de la participación educativa y su importancia para la mejora escolar. Para esto, se toman en evidencia diversas revisiones bibliográficas y reflexiones con el fin de contribuir al estudio sobre liderazgo y gestión educativa. En función de lo anterior, se analizará en primer lugar; la participación y su importancia para la mejora; en segundo lugar, la participación y el rol de la gestión directiva en generar esos procesos; tercero, la participación y la política educativa, tomando en consideración el Política Nacional de Convivencia Escolar y el Plan de Formación Ciudadana con fin de visualizar el impacto y la importancia de generar acciones que promuevan la participación educativa; finalmente, se realiza una reflexión a partir del desafío que significa instalar acciones participativas.

Un elemento a considerar, es que la participación escolar está fuertemente asociada al concepto de participación ciudadana (Ascorra, López \& Urbina, 2016) y/o participación democrática (Tonon, 2012) en 
Loyola, C. D. (2020). La importancia de la participación educativa en los procesos de mejora.

Revista Convergencia Educativa 7, julio, 1-18. DOI: http://doi.org/10.29035/rce.7.1

tanto se espera que en la escuela los estudiantes puedan realizar el ejercicio de la ciudadanía, a través de su involucramiento activo en los procesos escolares y también a través de la convivencia escolar (Martínez, Silva, Morandé, \& Canales, 2010, Schulz \& Fraillon, 2012, De Alba, García \& Santisteban, 2012). Es por esto, que la participación puede ser una práctica que no se presenta de la misma manera en todos los establecimientos, donde algunos atribuyen la participación a la promoción de la convivencia escolar y otros a la creación de instrumentos de gestión como el Proyecto Educativo Institucional (PEI) (Mineduc, 2015).

\subsection{METODOLOGÍA}

La propuesta metodológica del presente trabajo se fundamenta a partir de la revisión bibliográfica especializada en función del objetivo del ensayo. A continuación, se presentan los trabajos académicos (fuentes secundarias) más relevantes para el análisis (Tabla 1).

Tabla 1

Trabajos consultados.

DOCUMENTOS

\section{IMPORTANCIA EN FUNCIÓN DEL OBJETIVO}

1. Ministerio de Educación (2016). Gestión de la Documento ministerial, en donde se enfatiza la participación de la comunidad escolar. Dimensión: importancia de la participación de la comunidad escolar y Gestionando la convivencia y la participación de la funciona como una herramienta de gestión a partir de la comunidad escolar. reflexión de acciones o prácticas participativas.

2. Ministerio de Educación (2017). Orientaciones para Corresponde a un recurso elaborado por la División de la participación de las Comunidades Educativas en el Educación General. Este documento está asociado a la Marco del Plan de Formación Ciudadana. gestión de los espacios, condiciones y estructuras que se presentan para la participación.

3. Ministerio de Educación. (2019). Política Nacional de Indicaciones, objetivos y lineamientos centrales de la Convivencia Escolar (PNCE). política de convivencia escolar.

4. Ministerio de Educación (s.f.). ¿Por qué un plan de Documento presentado por Mineduc vinculado a la formación ciudadana en la escuela?

Ley 20.911 resaltando objetivos e importancia para los establecimientos educacionales.

5. Ascorra, P., López, V. y Urbina, C. (2016). Participación estudiantil en escuelas chilenas con buena y mala convivencia escolar.

Artículo que estudia la participación escolar tomando en consideración a los estudiantes a partir de realización de entrevistas. Estudia el concepto de participación estudiantil como de convivencia escolar.

6. Aravena, F., Escare, K. y Ramírez, J. (2019). La Artículo presentado como una nota técnica vinculado a participación democrática y la voz de los estudiantes: ¿Qué pueden hacer los líderes escolares? entregar lineamientos y actividades a desarrollar en el establecimiento educacional en función de la participación democrática.

7. Fierro, M. C. (2013). Convivencia inclusiva y democrática. Aborda temas asociados a la seguridad escolar y el rol Una perspectiva para gestionar la seguridad escolar. que cumplen los distintos estamentos educativos en esta acción. 
Loyola, C. D. (2020). La importancia de la participación educativa en los procesos de mejora.

Revista Convergencia Educativa 7, julio, 1-18. DOI: http://doi.org/10.29035/rce.7.1

8. Ley 20.911 (2016). Crea el plan de Formación Lineamientos legales asociados a la creación de los planes Ciudadana para los establecimientos educacionales de formación ciudadana. Objetivos y acciones a desarrollar. reconocidos por el Estado.

9. Ley $\mathrm{N}^{\circ} 20370$ (2009). Ley General de Educación. Lineamientos legales y generales que rigen la educación.

10.- Anderson, M. (2012). The Struggle for Collective Artículo que argumenta sobre el liderazgo colectivo, como Leadership: Thinking and Practice in a Multicampus distribuido o compartido que fortalecen a la educación School Setting. inclusiva.

11. Bellei, C., Valenzuela, J., Vanni, X., \& Contreras, D. (2014). Libro publicado por el Centro de Investigación Avanzada Lo aprendí en la escuela: ¿Cómo se logran procesos de en Educación (CIAE) y editorial LOM que ahonda en los mejoramiento escolar? procesos de mejora escolar tomando en consideración el liderazgo educativo y su diversas dimensiones e impactos.

12. De Alba, N., García, F., y Santisteban, A. (2012). Educar Libro con diversos artículos vinculado a una serie de para la participación ciudadana en la enseñanza de las dimensiones asociados a la ciudadanía en las Ciencias ciencias sociales. Sociales. Se destacan estudios de didáctica en educación ciudadana.

13. Hart, R. A. (1993). Children's participation: From Estudio que clasifica los distintos tipos/niveles de tokenism to citizenship. participación escolar

14. Henríquez, R., y Rasse, C., (2018). "Desafíos de la Artículo vinculado a la Ley 20.911 y sus desafíos para la Formación Ciudadana para su Enseñanza y Aprendizaje". comunidad educativa.

15. Cerda, A., Egaña, M., Magendzo, A., Santa Cruz, E., Libro que ahora diversos estudios asociados a la Varas, R., (2004). El Complejo Camino de la Formación Formación Ciudadana. ciudadana.

16. Leithwood, K.A, \& Riehl, C. (2003). What do we already Artículo que estudia la relación entre los líderes educativos know about successful school leadership? y la comunidad educativa a partir de la colaboración y apoyo mutuo.

17. Muñoz, G. (2011). La democracia y la participación en Artículo que establece una mirada crítica sobre las la escuela: ¿Cuánto se ha avanzado desde la normativa distintas políticas de participación establecidas desde la para promover la participación escolar? recuperación de la democracia por los gobiernos de la Concertación en el ámbito escolar.

18. Spillane, J. (2006). Distributed leadership.

Artículo que entrega información sobre el liderazgo distribuido. Características e importancia.

19. Tonon, G. (2012). Young people's quality of life and Libro vinculado el estudio de los jóvenes escolares y construction of citizenship. cómo influye la educación en la construcción de futuros ciudadanos.

20. Trilla, J. y Novella, A. (2001). Educación y participación Artículo en donde se analizan distintas formas de social de la infancia. participación de manera gradual en los ambientes educativos.

21. Loyola, C. (2019). Gestionando desde dentro la Proyecto de Tesis de magister asociado a gestión de Formación Ciudadana. la formación ciudadana y la gestión del cambio en la comunidad educativa.

22. Ministerio de Educación (2015). Marco para la Buena Dirección y el Liderazgo Escolar.

Documento Ministerial asociado a las dimensiones para la Dirección Escolar.

Fuente: Elaboración propia. 
Loyola, C. D. (2020). La importancia de la participación educativa en los procesos de mejora.

Revista Convergencia Educativa 7, julio, 1-18. DOI: http://doi.org/10.29035/rce.7.1

\section{PARTICIPACIÓN Y GESTIÓN DIRECTIVA}

Es importante que el ejercicio del liderazgo efectivo en las escuelas tenga una relación directa con la participación, cumpliendo un rol de refuerzo de los principios democráticos sobre la toma de decisiones en la organización escolar (Fusarelli, Kowalski y Petersen, 2011). De acuerdo con lo establecido por Trilla \& Novella (2001) se proponen distintos tipos de participación entre las que se destacan: participación simple, participación consultiva, participación proyectiva y metaparticipación. Los autores establecen que a medida que se avanza en estos niveles, va aumentando la complejidad de la participación (Tabla 2).

Tabla 2

Tipos de Participación.

Meta Participación En este tipo de participación son las personas quienes solicitan un cambio. Es decir, consideran que sus derechos participativos no son escuchados.

$\begin{array}{ll}\text { Participación Proyectiva } & \begin{array}{l}\text { Se caracteriza por el desarrollo de una participación activa, en que la o el sujeto escolar es } \\ \text { agente de las acciones y se involucra de manera permanente en el desarrollo de las mismas. } \\ \text { Señalan que en la participación proyectiva las ideas planteadas por la o el adulto sufren } \\ \text { modificaciones, siendo la distorsión de la idea original una manifestación de este tipo de } \\ \text { participación. }\end{array}\end{array}$

Participación Consultiva

Se caracteriza por estimular la participación. Se escucha la opinión de las y los sujetos, pudiendo ser esta última vinculante o no. Es decir, existirían dos caminos para este tipo de participación.

Participación Simple Se caracteriza por una participación que es medida en cantidad.

Fuente: Información obtenida a partir de Aravena, Escare y Ramírez (2019) y Mineduc (2017).

Es importante comprender que un proceso participativo necesita del diálogo y de generar espacios de reflexión crítica, que permita deconstruir significados que están arraigados en la cultura de las organizaciones y en los propios sujetos (Muñoz, 2011), es ahí la importancia de la gestión directiva.

El trabajo en la escuela no puede ser considerado como un proceso individual, este se encuentra en la obligación permanente de establecer interacciones entre los diversos actores de la comunidad escolar para poder cumplir con las distintas demandas que el cotidiano impone. Desde una perspectiva del liderazgo distribuido, se trasciende de la figura del director y apunta a los distintos actores que, de una u otra forma, ejercen funciones de influencia en la organización escolar. Estos múltiples actores capaces de influir y desplegar prácticas de liderazgo van desde jefes técnicos, coordinadores de área o docentes (Anderson, 2012, Crawford, 2012, Spillane, 2006, Spillane, Diamond \& Jita, 2003). Queda claro que no es sólo la visión del director lo que influye en la promoción de cambios profundos dentro de la escuela, la participación entonces cobra un valor como una práctica que facilita la instalación del liderazgo distribuido.

Por consiguiente, 
Loyola, C. D. (2020). La importancia de la participación educativa en los procesos de mejora.

Revista Convergencia Educativa 7, julio, 1-18. DOI: http://doi.org/10.29035/rce.7.1

...un establecimiento que sabe escuchar a sus docentes, asistentes de la educación, estudiantes y familias, y que genera diversas instancias de participación no se crea espontáneamente o por mero decreto, "se conforma en la dinámica de las relaciones humanas que se establecen" (Mineduc, 2015, 18, Mineduc, 2016).

Por lo mismo, definir e intencionar los procesos de participación es fundamental dentro de las prácticas del liderazgo directivo. Sin duda que el rol de establecer visiones compartidas en las comunidades académicas es fundamental para la administración y el liderazgo educativo. En este sentido, Castañeda \& Fernández (2007) resaltan lo importante que es tener una visión compartida para generar un aprendizaje organizacional y mayor participación de todos los actores de la comunidad educativa. "Involucrar a los distintos actores de la comunidad escolar: estudiantes, familia, docentes y asistentes de la educación, etc. facilita el trabajo en conjunto para alcanzar los objetivos, fortaleciendo la visión del establecimiento educacional" (Mineduc, 2016, 3). La importancia fundamental de establecer objetivos en una organización educativa queda establecida en lo señalo por Bellei et al. (2014) donde afirman que la definición de objetivos claros es el eje articulador de todas las decisiones de cambios impulsados por los directivos, y un elemento clave en el alineamiento de los esfuerzos de la comunidad escolar. Al respecto el Mineduc recomienda;

...los espacios donde la participación de distintos actores es valorada y considerada en la toma de decisiones favorecen la generación de un clima escolar positivo y los sentimientos de pertenencia de las personas a una comunidad.

De manera organizada, el director y su equipo directivo pueden promover la participación de los actores de la comunidad educativa mediante el trabajo efectivo del Consejo Escolar, el Consejo de Profesores, el Centro de Padres y Apoderados y el Centro de Estudiantes, entre otros (Mineduc, 2016,3).

Sin embargo, estos espacios de "participación" no deben estar sujeto a un modo de participación clientelista (Muñoz, 2011).

El Mineduc (2016) inspirados en el trabajo de Hart (1993) desarrolló distintos niveles de participación en el ámbito educativo. Cabe destacar que el hecho de que existan acciones o instancias participativas, éstas no aseguran necesariamente "la participación de todos los actores de la comunidad escolar o que estos puedan participar de distintas formas, no solo a nivel informativo, consultivo, sino que también a nivel propositivo y resolutivo" (Mineduc, 2016). A continuación, se presentan los cuatro niveles de participación en el ámbito escolar. 
Loyola, C. D. (2020). La importancia de la participación educativa en los procesos de mejora.

Revista Convergencia Educativa 7, julio, 1-18. DOI: http://doi.org/10.29035/rce.7.1

Tabla 3

Niveles de participación educativa.

\begin{tabular}{|c|c|c|c|c|}
\hline $\begin{array}{l}\text { NIVELES DE } \\
\text { PARTICIPACIÓN }\end{array}$ & 1. INFORMATIVO & 2. CONSULTIVO & 3. PROPOSITIVO & 4. RESOLUTIVO \\
\hline Descripción & $\begin{array}{l}\text { El director y su } \\
\text { equipo directivo } \\
\text { entregan } \\
\text { información a } \\
\text { los actores de la } \\
\text { comunidad. }\end{array}$ & $\begin{array}{l}\text { El director y su } \\
\text { equipo directivo } \\
\text { consultan a los } \\
\text { actores involucrados } \\
\text { antes de tomar ellos } \\
\text { las decisiones }\end{array}$ & $\begin{array}{l}\text { El director y su } \\
\text { equipo directivo } \\
\text { están abiertos a } \\
\text { recibir y tomar } \\
\text { en consideración } \\
\text { ideas y propuestas } \\
\text { nuevas, así como } \\
\text { acciones concretas } \\
\text { de ejecución desde } \\
\text { distintos actores. } \\
\text { La diferencia } \\
\text { respecto al nivel } \\
\text { consultivo es que } \\
\text { los actores toman } \\
\text { la iniciativa en } \\
\text { participar. }\end{array}$ & $\begin{array}{l}\text { El director y su } \\
\text { equipo directivo, } \\
\text { junto con los actores } \\
\text { de la comunidad } \\
\text { escolar, toman } \\
\text { en conjunto las } \\
\text { decisiones finales. } \\
\text { La opinión de los } \\
\text { distintos actores es } \\
\text { igual de válida que } \\
\text { la del director y su } \\
\text { equipo. }\end{array}$ \\
\hline Objetivos & $\begin{array}{l}\text { Entregar } \\
\text { información } \\
\text { necesaria a los } \\
\text { actores de la } \\
\text { comunidad escolar } \\
\text { sobre determinados } \\
\text { temas, decisiones, } \\
\text { noticias, etc. }\end{array}$ & $\begin{array}{l}\text { Obtener la opinión } \\
\text { de los actores de la } \\
\text { comunidad escolar. }\end{array}$ & $\begin{array}{l}\text { Recibir ideas } \\
\text { y propuestas } \\
\text { concretas de } \\
\text { los actores de la } \\
\text { comunidad. }\end{array}$ & $\begin{array}{l}\text { Tomar decisiones } \\
\text { en conjunto con } \\
\text { la comunidad } \\
\text { escolar. Compartir } \\
\text { responsabilidades. }\end{array}$ \\
\hline Compromisos que implica & $\begin{array}{l}\text { El director } \\
\text { y su equipo } \\
\text { directivo están } \\
\text { comprometidos } \\
\text { a atenerse a la } \\
\text { información } \\
\text { entregada, a menos } \\
\text { que se vuelva } \\
\text { a entregar otra } \\
\text { información a } \\
\text { todos los actores } \\
\text { involucrados, } \\
\text { aclarando todos los } \\
\text { cambios. }\end{array}$ & $\begin{array}{l}\text { El director y su } \\
\text { equipo directivo } \\
\text { no están obligados } \\
\text { a ejecutar las } \\
\text { decisiones } \\
\text { sugeridas; pueden } \\
\text { incorporar algunas } \\
\text { ideas y dejar de lado } \\
\text { otras. Se trata de la } \\
\text { manifestación de la } \\
\text { voluntad general o } \\
\text { popular de forma no } \\
\text { vinculante. }\end{array}$ & $\begin{array}{l}\text { El director y su } \\
\text { equipo directivo } \\
\text { están obligados a } \\
\text { escuchar y evaluar } \\
\text { las sugerencias o } \\
\text { propuestas hechas } \\
\text { por los distintos } \\
\text { actores de la } \\
\text { comunidad sin } \\
\text { importar su rol o } \\
\text { función. }\end{array}$ & $\begin{array}{l}\text { El director y su } \\
\text { equipo directivo } \\
\text { se comprometen a } \\
\text { tomar la decisión } \\
\text { final en conjunto } \\
\text { con los actores } \\
\text { involucrados. }\end{array}$ \\
\hline
\end{tabular}

Fuente: extraído de Mineduc, 2016.

Al respecto,

"se espera es que el director y su equipo directivo no solo informen de las decisiones tomadas o consulten a distintos actores antes que ellos mismos las tomen; se espera que reciban propuestas y definan en conjunto las decisiones finales" (Mineduc, 2016). 
Loyola, C. D. (2020). La importancia de la participación educativa en los procesos de mejora.

Revista Convergencia Educativa 7, julio, 1-18. DOI: http://doi.org/10.29035/rce.7.1

\section{PARTICIPACIÓN Y LA POLÍTICA EDUCATIVA}

Como ya lo hemos mencionado, "participar consiste en el involucramiento y colaboración de las y los integrantes de la comunidad educativa en un marco de respeto, permitiendo que la gestión global de la misma cuente con mayores niveles de legitimidad" (Mineduc, 2017, 15). En el caso de las políticas educativas, estas han estado en permanente evolución vinculada a los cambios de nuestro entorno social, cultural, político, económico e institucional. Así, mientras que en el siglo pasado la toma de decisiones era exclusivamente estatal, con su poder de crear, estructurar y modificar el ciclo de las políticas públicas, en la actualidad la participación de diversos actores, la creación de consenso y la legitimidad en los asuntos públicos, se han tornado ineludibles para la gobernanza en cualquier territorio (Lahera, 2008, Espinoza, 2009, Aziz, 2018). En ese sentido, las políticas públicas son instrumentos de gobernabilidad que dan forma a la política, asignando ganadores y perdedores entre ciudadanos y grupos de interés, lo que suele afectar el ciclo de política, la participación y las demandas futuras (May \& Jochim, 2013). En el caso de Chile, esta participación de distintos actores en los marcos legales de la educación tiene su inicio en el año 2006 con mayor preponderancia con marchas estudiantiles y el involucramiento de la sociedad en general. A partir de esa fecha se ha iniciado un periodo de participación de actores claves en la elaboración de las políticas, lo que ha influenciado varias leyes fundamentales que dan base a una reforma estructural del sistema educativo chileno, como la Subvención Escolar Preferencial (SEP), la Ley General de Educación (LGE), la Ley de Aseguramiento de la Calidad (SAC), la Ley de Inclusión, la Ley de Carrera Docente y la Ley que crea el nuevo Sistema de Educación Pública (Aziz, 2018), la Política Nacional de Convivencia Escolar (PNCE) y el desarrollo de la Formación Ciudadana (PFC), entre otras.

A continuación, se analizará la Política Nacional de Convivencia Escolar (PNCE) y el Plan de Formación Ciudadana (PFC) como procesos de participación activa por parte de los diversos actores de la comunidad educativa.

\subsection{Política Nacional de Convivencia Escolar (PNCE)}

La reciente Política Nacional de Convivencia Escolar define la convivencia escolar como "el conjunto de las interacciones y relaciones que se producen entre todos los actores de la comunidad" (PNCE, 2019, 9, citado en Aravena et al., 2019). "Dentro de este marco, se establece que la convivencia es un proceso permanente de aprendizaje y enseñanza, y una construcción sociocultural, de carácter dinámico y cotidiano" (Aravena et al., 2019, 2). Sin duda, se espera que esta actualización de la política de convivencia escolar modifique los procesos de toma de decisión a partir de la participación de todos sus actores. Al respecto;

...se espera de esta política sea una herramienta que aporte a la gestión de la convivencia y a "una toma de conciencia sobre la materia de todos los miembros de la comunidad educativa: equipos 
directivos, docentes, asistentes de la educación, padres y apoderados y, por supuesto, los más de 3,5 millones de estudiantes que existen en Chile (PNCE, 2019: 7).

Es interesante recalcar que la PNCE tiene cuatro características que permiten la promoción de la convivencia escolar en los establecimientos educacionales.

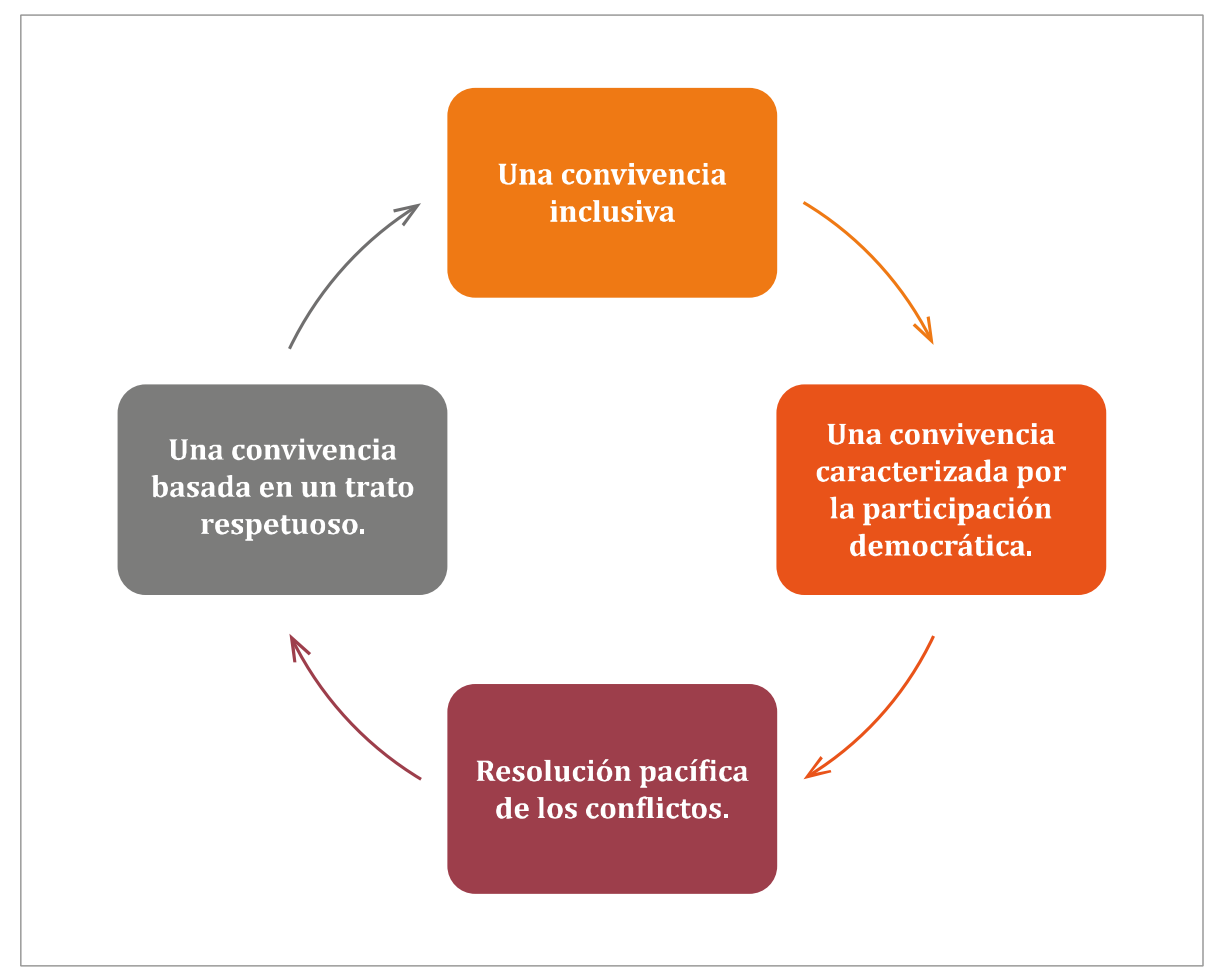

Figura 1. Ejes de la Política Nacional de Convivencia Escolar.

Fuente: Elaboración propia a partir de PNCE (2019).

Desde el punto de vista de la participación, "se busca promover la colaboración que faciliten la construcción de un sentido de pertenencia basado en una identificación positiva con la comunidad educativa, su cultura y las actividades que en ella se realizan" (PNCE, 2019, 14). Alude también a la existencia de oportunidades para la expresión de opiniones, la deliberación y el debate fundamentado de ideas, la construcción de acuerdos y el respeto de estos por parte de toda la comunidad, y la colaboración necesaria para llevar adelante las distintas actividades del quehacer cotidiano (PNCE, 2019). Es de vital importancia la labor y rol que juega el Encargado de Convivencia Escolar en los establecimientos educacionales quién debe ejercer una función de "Sponsor" liderando y gestionando el cambio a partir de estos ejes establecidos en la política.

La gestión debe ser coherente y pertinente, pues busca unir las declaraciones conceptuales, el tipo de convivencia que se quiere promover y las características del contexto específico de cada 
Loyola, C. D. (2020). La importancia de la participación educativa en los procesos de mejora.

Revista Convergencia Educativa 7, julio, 1-18. DOI: http://doi.org/10.29035/rce.7.1

establecimiento y sus actores, con los objetivos y acciones que se diseñen e implementen (PNCE, 2019, 16; ver también a Fierro, 2013, Mineduc, 2015).

El desafío, por tanto, es propiciar, dinamizar y permitir el funcionamiento de estas instancias de manera de constituirse en comunidades que se gestionan y se recrean en un proceso participativo de autoconstrucción (Mineduc, 2017).

\subsection{Plan de Formación Ciudadana (PFC)}

La participación en el aula debe ser vital para el desarrollo ciertos aprendizajes en ciudadanía. Estas prácticas escolares deben ser entes colaboradores en la enseñanza - aprendizaje y en la interacción (Mineduc, 2017). Al respecto fortalecer preguntas reflexivas dentro del aula, Lluvia de ideas, definir con propias palabras, participar vía votos, expresar sentimientos y afectos, solicitar opiniones personales, elaborar pensamientos complejos, que los estudiantes propongan formas de trabajo, son algunas de las estrategias que tienen buenos resultados. Del mismo modo, la reflexión es una capacidad fundamental en el ser humano que permite ser partícipe de incidir en los cambios que ocurren en la sociedad. Al respecto, las prácticas reflexivas deben ir encaminadas a planificar las actividades escolares considerando secuencias reflexivas, genera clima de aula, fomentar el debate, incluir al otro, reflexionar sobre experiencias personales o familiares, reflexionar sobre la materia, reflexión sobre lo comunitario, reflexionar sobre la sociedad, los medios de comunicación, entre otros aspectos.

Gracias a la Ley 20.911 se posiciona en el currículum la inclusión de la Formación Ciudadana. Llama la atención de la Ley la amplitud de sus propósitos. "Promover" y “Fomentar” son los propósitos más utilizados para dar sentido a un conjunto de significados ya presentes en nuestros currículos: ciudadanía, derechos y deberes, Estado de Derecho, instituciones, virtudes cívicas, derechos humanos, Constitución Política, tratados internacionales, derechos del niño, diversidad, interés en lo público y cultura democrática (Henríquez y Rasse, 2018).

En este contexto, la Ley 20.911 que crea el Plan de Formación Ciudadana invita a las escuelas y liceos a intencionar este diálogo. Para ello, establece que:

Los establecimientos educacionales reconocidos por el Estado deberán incluir en los niveles de enseñanza parvularia, básica y media un Plan de Formación Ciudadana, que integre y complemente las definiciones curriculares nacionales en esta materia, que brinde a los estudiantes la preparación necesaria para asumir una vida responsable en una sociedad libre y dé orientación hacia el mejoramiento integral de la persona humana, como fundamento del sistema democrático, la justicia social y el progreso. Asimismo, deberá propender a la formación 
Loyola, C. D. (2020). La importancia de la participación educativa en los procesos de mejora.

Revista Convergencia Educativa 7, julio, 1-18. DOI: http://doi.org/10.29035/rce.7.1

de ciudadanos, con valores y conocimientos para fomentar el desarrollo del país, con una visión del mundo centrada en el ser humano, como parte de un entorno natural y social. En el caso de la educación parvularia, este plan se hará de acuerdo a las características particulares de este nivel y su contexto, por ejemplo, a través del juego (Mineduc, s.f., 8)

Con el fin de alcanzar lo que establecen los siguientes objetivos a desarrollar en las escuelas y liceos:

a) Promover la comprensión y análisis del concepto de ciudadanía y los derechos y deberes asociados a ella, entendidos éstos en el marco de una república democrática, con el propósito de formar una ciudadanía activa en el ejercicio y cumplimiento de estos derechos y deberes. b) Fomentar en los estudiantes el ejercicio de una ciudadanía crítica, responsable, respetuosa, abierta y creativa. c) Promover el conocimiento, comprensión y análisis del Estado de Derecho y de la institucionalidad local, regional y nacional, y la formación de virtudes cívicas en los estudiantes. d) Promover el conocimiento, comprensión y compromiso de los estudiantes con los derechos humanos reconocidos en la Constitución Política de la República y en los tratados internacionales suscritos y ratificados por Chile, con especial énfasis en los derechos del niño. e) Fomentar en los estudiantes la valoración de la diversidad social y cultural del país. f) Fomentar la participación de los estudiantes en temas de interés público.g) Garantizar el desarrollo de una cultura democrática y ética en la escuela. h) Fomentar una cultura de la transparencia y la probidad. i) Fomentar en los estudiantes la tolerancia y el pluralismo (Ley 20.911, 2016).

Uno de los aspectos relevantes es la participación de la comunidad educativa. De acuerdo con Cerda et al. (2004) es necesario que se establezcan dinámicas de discusión sobre formación ciudadana en el establecimiento educacional asociadas a: a) el imperativo del cambio, b) necesidad de introducir una mirada ética en la formación ciudadana, c) importancia de lo público, d) expandir la mirada crítica, e) el currículo y las prácticas, f) convertir las prácticas cotidianas en motivo de reflexión y observación para estudiantes y profesores.

Por otro lado, no se debe caer en malas interpretaciones teniendo en cuenta que realizar un Plan de Formación Ciudadana en la escuela no garantiza que se esté realmente promoviendo el desarrollo de la Formación Ciudadana de una manera íntegra. No consiste solamente en crear un instrumento que sirva para rendir cuentas (acountability) sino que realmente permita desarrollar y formar ciudadanos activos, críticos, sociales y políticos (Loyola, 2019).

Finalmente, es necesario que los equipos directivos, tanto en su gestión y liderazgo no establezca limitaciones institucionales, éstas asociadas a políticas de rendición de cuenta, ya que impondrían a los docentes mayor carga de tiempo y escasos momentos de reflexión y participación escolar. De ahí la importancia de tomar decisiones acordes a los objetivos planeados en el Proyecto Educativo Institucional. 
Desde entonces, se están realizando un conjunto de acciones y actividades, con fuerte protagonismo de la comunidad escolar. Esto implica la formación de docentes y directivos, actividades extracurriculares, iniciativas de apertura a la comunidad y "una planificación curricular que visibiliza los objetivos de aprendizaje transversales que refuerzan el desarrollo de la ciudadanía, la ética y la cultura democrática en las diversas asignaturas" (Ley 20.911).

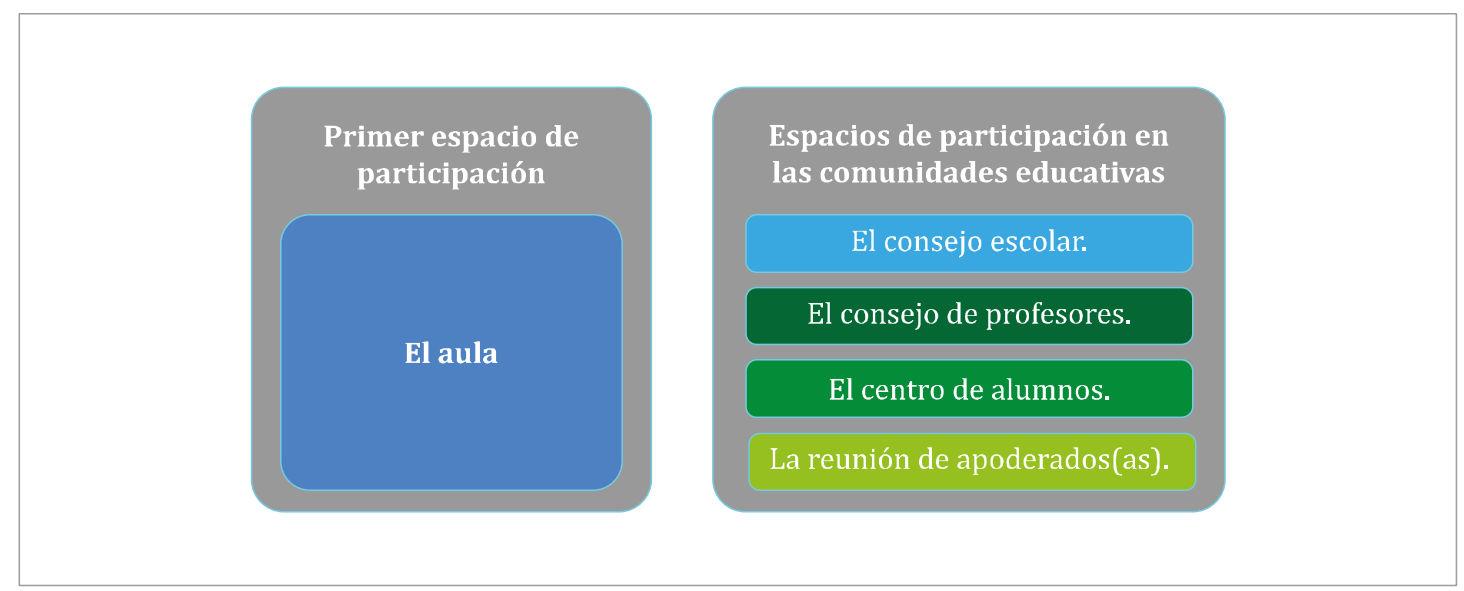

Figura 2. Espacios de participación existentes en la unidad educativa. Fuente: elaboración propia.

Según texto del Ministerio de Educación, en relación a los espacios de participación;

...para lograr esto, los establecimientos deben promover y fortalecer espacios reales de encuentro en que se respeten los aportes, experiencias y atribuciones del rol que le compete a cada actor. No solo se espera que se fortalezcan y se establezcan espacios formales de participación como los nombrados anteriormente (Consejo Escolar, Consejo de Profesores, Centro de Padres y Apoderados, Centro de Estudiantes), sino que esto incluya también espacios informales, como actividades recreacionales, deportivas y artísticas, entre otras instancias (Mineduc, 2016).

A raíz de lo anterior, presentamos siete acciones que fomentan la participación en el ámbito escolar donde, especialmente los líderes educativos, deben promover y desarrollar. 


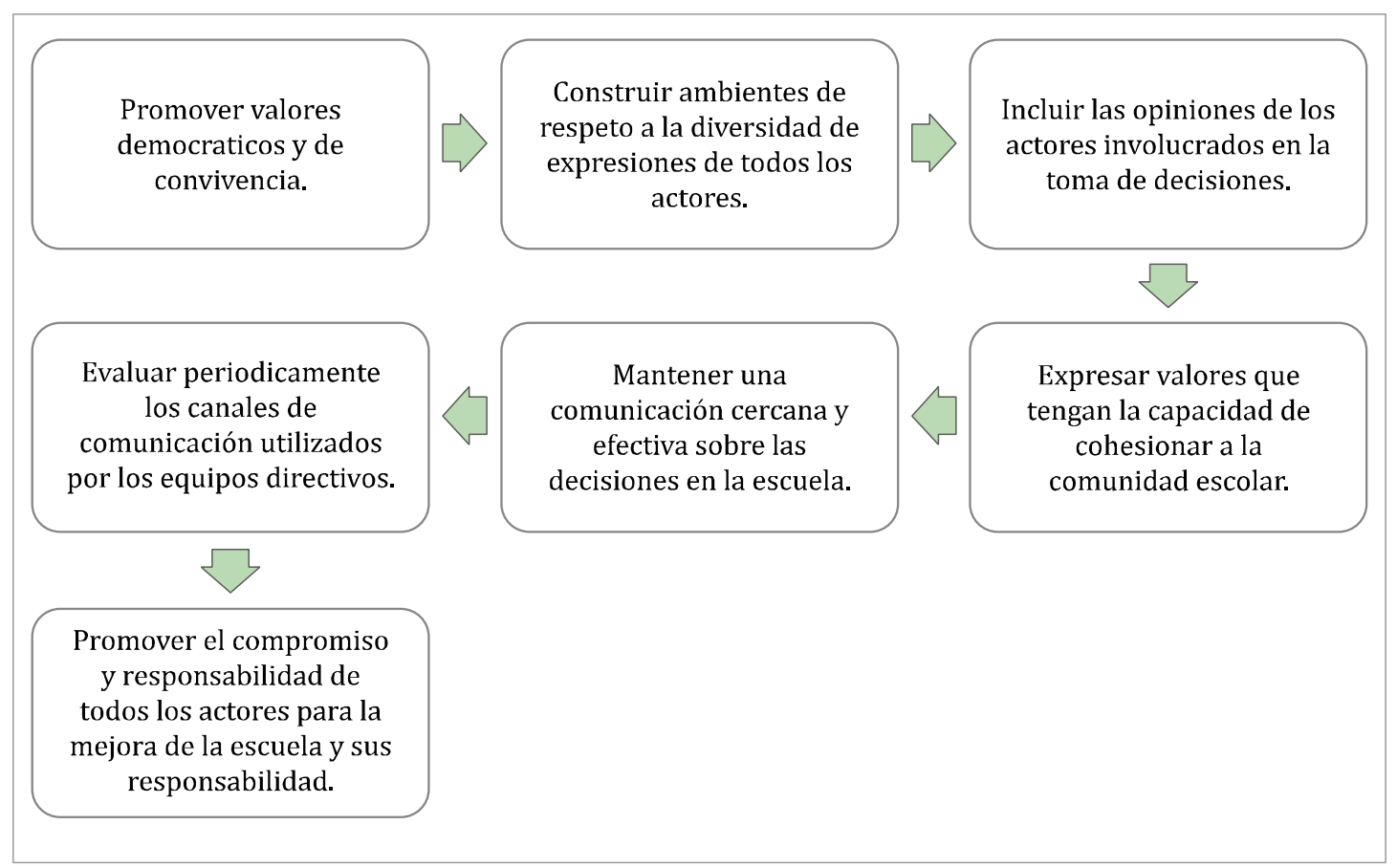

Figura 3. Prácticas que fomentan la participación en la escuela. Fuente: elaboración propia.

El establecer valores de convivencia y que estos sean democráticos permitirá que los distintos actores de la comunidad escolar se sientan en confianza (relacional) para participar en las diversas actividades y acciones que pueden surgir a partir del PEI, PME, PFC, PNC, entre otras. El Proyecto Educativo Institucional representa, desde sus propósitos, una de las mayores instancias de participación y representatividad de entre otros tópicos el de una visión compartida, promoción de prácticas democráticas y el fortalecimiento de una sana convivencia entre todos los integrantes. Por esta razón se vuelve relevante poder analizar la función que este instrumento cumple en el proceso de mejora.

Es fundamental, establecer normas de respeto cuidando el ambiente y las diversas expresiones, evitando caer en la discriminación, aislamiento, bullying, etc. Para que exista una participación efectiva se debe promover la comunicación fluida y tomar en consideración todas las voces de los actores educativos. La cooperación y colaboración son elementos que van de la mano con la participación, esto permite la cohesión y sinergia en la comunidad escolar. Sin duda, que el monitoreo es esencial, desde la perspectiva de la gestión, para evaluar que las acciones y actividades están teniendo un impacto positivo en la organización escolar. Es importante recalcar que el compromiso y establecer metas y expectativas son esenciales para fomentar procesos de mejora escolar.

Vinculado con estas prácticas, se presenta una experiencia educativa en donde se evidencia la importancia de promover acciones participativas en la comunidad escolar, entre la que se destaca "el patio 
Loyola, C. D. (2020). La importancia de la participación educativa en los procesos de mejora.

Revista Convergencia Educativa 7, julio, 1-18. DOI: http://doi.org/10.29035/rce.7.1

rojo pedagógico" (Agencia de la Calidad, 2016). Consiste en la implementación de exposición de los estudiantes permite un proceso de aprendizaje complejo, mostrando lo que saben y desarrollando destrezas comunicativas variadas, tales como: representar, explicar y demostrar, entre otras.

Con esta actividad se logra fomentar la participación de todos los estudiantes, desarrollando habilidades comunicacionales y sociales de los estudiantes incorporando la presencia activa de apoderados en el proceso de aprendizaje de los estudiantes. Esta dinámica permite desarrollar una evaluación de aprendizajes de manera no tradicional (Agencia de la calidad, 2016).

\section{CONCLUSIONES}

Se aprecia un esfuerzo por parte del Ministerio de Educación en la Política Nacional de Convivencia Escolar y en la Formación Ciudadana, donde se pretende fomentar la participación en la comunidad escolar tomando en consideración a todos los actores de la comunidad educativa. Sin embargo, el generar una participación activa genera un desafío potente para los líderes educativos en movilizar creencias, asociadas a la colaboración mutua entre los que están inserto en la organización escolar.

La implicancia de este ensayo está estrechamente ligado al desafío asociado a la participación. Este desafío está en no plantear la participación educativa a partir de marcos normativos "impuestos" sea esta una ley o decreto, sino que, por el contrario, las comunidades educativas busquen la promoción de acciones colaborativas - participativas a partir de la autogestión, tomando sus contextos y criterios, favoreciendo a la creación de altas expectativas, confianza y procesos de mejora.

Entendemos que la participación educativa corresponde a una herramienta para la mejora escolar muy significativa. Así lo afirma y demuestra la evidencia especializada. De ahí surge la necesidad constante de crear, innovar, y establecer espacios de participación en la comunidad educativa tal como lo hicieron en la experiencia educativa "El patio rojo pedagógico", donde se ponen en práctica cuestiones teóricas materializando acciones que generan impacto en la comunidad educativa. Los resultados y logros están relacionados con la creación de espacios de confianza entre equipo directivo, docentes, estudiantes y apoderados; colaboración y participación de las familias; hay un valor de las iniciativas y potencia la articulación de las asignaturas. La participación consiste también en un proceso formativo de enseñanza aprendizaje a través del cual se puede facilitar la construcción de valores éticos y democráticos para la formación ciudadana. La consolidación de unos principios éticos nos conduce a pensar en una ética democrática donde el ciudadano participa en la transformación de su medio (Muñoz, 2011). Al respecto, Freire (2002), afirma que nos sentimos parte de un conflicto cuando la participación se da en condiciones de igualdad, cuando profundizamos en el diálogo y cuando analizamos y revisamos la realidad para posteriormente transformarla. En este sentido el diálogo supone fortalecer las condiciones de igualdad 
Loyola, C. D. (2020). La importancia de la participación educativa en los procesos de mejora.

Revista Convergencia Educativa 7, julio, 1-18. DOI: http://doi.org/10.29035/rce.7.1

entre las personas que lo integran, pero se necesita asegurar unas condiciones para construir consenso en dicho diálogo.

Es necesario establecer diversos canales de participación en la escuela, en donde se establezcan instancias efectivas, tanto de comunicación como de colaboración. Si bien es cierto, cada establecimiento educacional posee asociaciones formales de participación, que, sin duda, deben ser eficientes, también se debe mirar y visualizar aquellas instancias informales de participación con el objetivo de buscar distintas metodologías para recoger apreciaciones y voces de todos(as) los actores de la comunidad educativa. Para esto, se sugiere tomar en consideración, los tipos y niveles de participación que se dan en un establecimiento educacional, como también, las prácticas y acciones que se deben tomar en consideración para establecer procesos de mejora escolar.

\section{REFERENCIAS BIBLIOGRÁFICAS}

Agencia de la Calidad (2016). Experiencia educativa Patio Rojo pedagógico [video]. Recuperado de https://www.agenciaeducacion.cl/multimedia/visitas-aprendizaje-compartiendo-experienciaseducativas/

Anderson, M. (2012). The Struggle for Collective Leadership: Thinking and Practice in a Multicampus School Setting. Educational Management Administration \& Leadership, 40(3), 328-342. DOI: https://doi.org/10.1177/1741143212436955

Aravena, F., Escare, K., \& Ramírez, J. (2019). La participación democrática y la voz de los estudiantes: ¿Qué pueden hacer los líderes escolares? Nota Técnica $\mathrm{N}^{\circ} 2$. Valparaíso: Lideres Educativos, Centro de Liderazgo para la Mejora Escolar. Recuperado de https://www.lidereseducativos.cl/wp-content/uploads/2019/06/NT2_L1_F.A._LA-PARTICIPACION-DEMOCRATICA-Y-LA-VOZ-DE-LOS-ESTUDIANTES_26-06-19.pdf

Ascorra, P., López, V., \& Urbina, C. (2016). Participación estudiantil en escuelas chilenas con buena y mala convivencia escolar. Revista de Psicología, 25(2), 1-18. Recuperado de https://scielo.conicyt.cl/scielo.php?script=sci arttext\&pid=S0719-05812016000200003\&lng=es\&tlng=es.

Aziz, C. (2018). Evolución e implementación de las políticas educativas en Chile. Nota técnica $\mathrm{N}^{\circ} 2$. Valparaíso: Lideres Educativos, Centro de Liderazgo para la Mejora Escolar. Recuperado de https://www.lidereseducativos.cl/wp-content/uploads/2018/06/NT2 L6 C.A Evolucio\%CC\%81n-e-implementacio\%CC\%81n-de-las-poli\%CC\%81ticas-educativas-en-Chile.pdf 
Loyola, C. D. (2020). La importancia de la participación educativa en los procesos de mejora.

Revista Convergencia Educativa 7, julio, 1-18. DOI: http://doi.org/10.29035/rce.7.1

Bellei, C., Valenzuela, J., Vanni, X., \& Contreras, D. (2014). Lo aprendí en la escuela: ¿Cómo se logran procesos de mejoramiento escolar? Santiago: LOM.

Castañeda, D. I., \& Fernández, M. (2007). Validación de una escala de niveles y condiciones de aprendizaje organizacional. Universitas Psychológica, 6(2), 245-254. Recuperado de http://www.scielo.org.co/pdf/rups/v6n2/v6n2a04.pdf

Cerda, A., Egaña, M., Magendzo, A., Santa Cruz, E., \& Varas, R. (2004). El Complejo Camino de la Formación ciudadana. Santiago: LOM.

Crawford, M. (2012). Solo and Distributed Leadership: Definitions and Dilemmas. Educational Management Administration \& Leadership, 40(5), 610-620. DOI: https://doi.org/10.1177/1741143212451175

De Alba, N., García, F., \& Santisteban, A. (2012). Educar para la participación ciudadana en la enseñanza de la ciencias sociales. Sevilla: Asociación Universitaria de Profesorado de Didáctica de las Ciencias Sociales.

Espinoza, 0. (2009). Reflexiones sobre los conceptos de "política", políticas públicas y política educacional. Archivos Analíticos de Políticas Educativas, 17(8), 1-13. Recuperado de https://www.redalyc.org/pdf/2750/275019727008.pdf

Fierro, M. C. (2013). Convivencia inclusiva y democrática. Una perspectiva para gestionar la seguridad escolar. Sinéctica, (40), 1-18. Recuperado de http://www.scielo.org.mx/scielo.php?pid=S1665109X2013000100005\&script=sci_arttext\&tlng=en

Freire, P. (2002). Pedagogía de la esperanza. Un reencuentro con la pedagogía del oprimido. Argentina: Siglo XXI.

Fusarelli, I., Kowalski, T., \& Petersen, G. (2011). Distributive leadership, civic engagement, and deliberative democracy as vehicles for School Improvement. Leadership and Policy in Schools, 10(1), 43-62. DOI: https://doi.org/10.1080/15700760903342392

Hart, R. A. (1993). Children's participation: From tokenism to citizenship. Florence: Unicef, International Child Development Center.

Henríquez, R., \& Rasse, C. (2018). Desafíos de la Formación Ciudadana para su Enseñanza y Aprendizaje. En I. Sánchez (Ed.), Ideas en Educación II. Definiciones en tiempos de cambio. Santiago: Ediciones UC. Recuperado de http://ceppe.uc.cl/images/stories/libro/ideas-en-educacion-2.pdf

Lahera, E. (2008). Introducción a las políticas públicas. Santiago: Fondo de Cultura Económica. 
Loyola, C. D. (2020). La importancia de la participación educativa en los procesos de mejora.

Revista Convergencia Educativa 7, julio, 1-18. DOI: http://doi.org/10.29035/rce.7.1

Leithwood, K.A, \& Riehl, C. (2003). What do we already know about successful school leadership? Washington, DC: AERA Division A task force on developing research in educational leadership.

Ley $N^{\circ} 20.911$ (2016). Crea el plan de Formación Ciudadana para los establecimientos educacionales reconocidos por el Estado. Diario Oficial de la República de Chile. Santiago, Chile.

Ley Nº 20.370 (2009). Ley General de Educación. Diario Oficial de la República de Chile, Santiago, Chile.

Loyola, C. (2019). Gestionando desde dentro la Formación Ciudadana (Tesis de postgrado). Universidad Católica del Valparaíso, Valparaíso, Chile.

Martínez, M. L., Silva, C., Morandé, M., \& Canales, L. (2010). Los jóvenes ciudadanos: reflexiones para una política de formación ciudadana juvenil. Última Década, 32, 105-118. DOI: http://dx.doi.org/10.4067/S0718-22362010000100006

May, P. J., \& Jochim, A. E. (2013). Policy regime perspectives: Policies, politics and governing. Policy Studies Journal, 41(3). DOI: https://doi.org/10.1111/psj.12024

Ministerio de Educación (2015). Marco para la Buena Dirección y el Liderazgo Escolar. Recuperado de https://liderazgoescolar.mineduc.cl/wp-content/uploads/sites/55/2016/04/MBDLE_2015.pdf

Ministerio de Educación (2016). Gestión de la participación de la comunidad escolar. Dimensión: Gestionando la convivencia y la participación de la comunidad escolar. Recuperado de https://bibliotecadigital.mineduc.cl/handle/20.500.12365/582

Ministerio de Educación (2017). Orientaciones para la participación de las Comunidades Educativas en el Marco del Plan de Formación Ciudadana. Recuperado de https://formacionciudadana.mineduc.cl/wp-content/uploads/sites/46/2019/05/Orientaciones-para-la-participacion-de-las-comunidades-educativas-en-el-marco-del-PFC.pdf

Ministerio de Educación. (2019). Política Nacional de Convivencia Escolar (PNCE). Recuperado de http://convivenciaescolar.mineduc.cl/politica-nacional/

Ministerio de Educación (s.f.) ¿Por qué un plan de formación ciudadana en la escuela? Conversemos: Cuaderno Docente, 7, 3-13. División de Educación General. Recuperado de https://basica.mineduc.cl/wp-content/uploads/sites/25/2017/03/Libro-Conversemos-Formacion-Ciud-Escuela.pdf

Muñoz, G. (2011). La democracia y la participación en la escuela: ¿Cuánto se ha avanzado desde la normativa para promover la participación escolar? Revista de Estudios y Experiencias en Educación, 10(19), 107-129. Recuperado de https://www.redalyc.org/pdf/2431/243120126006.pdf 
Loyola, C. D. (2020). La importancia de la participación educativa en los procesos de mejora.

Revista Convergencia Educativa 7, julio, 1-18. DOI: http://doi.org/10.29035/rce.7.1

Schulz, W., \& Fraillon, J. (2012). Students' participation in and valuing of civic engagement at school. Trabajo presentado en Annual Meetings of the American Educational Research Association, Vancouver, Canadá. Recuperado de https://www.semanticscholar.org/paper/Students'-participation-in-andvaluing-of-civic-at-Schulz-Fraillon/8bee7b25bef5ae1e93ef325d5d69452be8e33165

Spillane, J. (2006). Distributed leadership. San Francisco: Jossey-Bass.

Spillane, J., Diamond, J., \& Jita, L. (2003). Leading instruction: the distribution of leadership for instruction. Journal of Curriculum Studies, 35(5), 533-543. DOI: 10.1080/0022027021000041972

Tonon, G. (2012). Young people's quality of life and construction of citizenship. Dordrecht: Springer.

Trilla, J., \& Novella, A. (2001). Educación y participación social de la infancia. Revista Iberoamericana de Educación, 26, 137-164. https://rieoei.org/RIE/article/view/982

UNICEF (2005). Participación de los Centros de Padres en la Educación: Ideas y Herramientas para mejorar la organización. Recuperado de https://www.researchgate.net/publication/303271940 Participacion de los Centros de Padres en la Educacion Expectativas Demandas Desafios y Compro$\underline{\text { misos }}$

\section{Datos de correspondencia}

Christian Danner Loyola Bustos

Magíster en Política Educativa.

Magíster en Liderazgo y Gestión de las Organizaciones Escolares.

Pontificia Universidad Católica de Valparaíso PUCV.

Santiago, Chile

Dirección postal: Daniel Durán 1576, Melipilla. RM. Santiago, Chile.

ORCID: https://orcid.org/0000-0003-4792-9936

Email: loyola.cristian.d@gmail.com 